Pure and Applied Mathematics Quarterly

Volume 7, Number 2

(Special Issue: In honor of

Frederick W. Gehring, Part 2 of 2$)$

425-453, 2011

\title{
Mappings and Spaces, 2
}

\author{
Stephen W. Semmes*
}

\begin{abstract}
This paper is concerned with analysis on metric spaces in a variety of settings and with several kinds of structure.

Keywords: Analysis on metric spaces, complex-analytic metric spaces, Poincaré inequalities
\end{abstract}

\section{Contents}

1 Basic notions

Received June 14, 2007.

*Some of this material was presented at the November, 2006 meeting of the American Mathematical Society at the University of Arkansas. I would like to thank participants of the meeting and other readers for their comments and suggestions, including the referee. As we celebrate Fred Gehring's 80th birthday, we also mourn the passing of Juha Heinonen, whose life and work are inspirations for us all. 


\section{Basic notions}

Let us begin with a brief review of some aspects of analysis on metric spaces. Let $(M, d(x, y))$ and $(N, \rho(u, v))$ be metric spaces, let $\alpha$ be a positive real number, and let $C$ be a nonnegative real number. A mapping $f: M \rightarrow N$ is said to be C-Lipschitz of order $\alpha$ if

$$
\rho(f(x), f(y)) \leq C d(x, y)^{\alpha}
$$

for every $x, y \in M$. For example, constant functions are 0-Lipschitz of every order and are the only 0-Lipschitz functions, and the identity mapping $f(x)=x$ is 1-Lipschitz of order 1 as a mapping from $M$ to $M$. If $f(x)$ is a continuouslydifferentiable real or complex-valued function on the real line, then $f(x)$ is $C$ Lipschitz of order 1 if and only if $\left|f^{\prime}(x)\right| \leq C$ for every $x \in \mathbf{R}$.

If $f_{1}, f_{2}$ are complex-valued functions on a metric space $M$ which are $C_{1}$, $C_{2}$-Lipschitz of order $\alpha$ for some $\alpha>0$ and $C_{1}, C_{2} \geq 0$, then it is easy to see that $f_{1}+f_{2}$ is $\left(C_{1}+C_{2}\right)$-Lipschitz of order $\alpha$. If $f$ is a complex-valued $C$-Lipschitz function of order $\alpha$ on $M$ and $a$ is a complex number, then $a f$ is $(|a| C)$-Lipschitz function of order $\alpha$. If $f_{1}, f_{2}$ are also bounded, then the product $f_{1} f_{2}$ is Lipschitz of order $\alpha$ too. The composition of a Lipschitz mapping of order $\alpha$ and a Lipschitz mapping of order $\beta$ is Lipschitz of order $\alpha \beta$. Lipschitz mappings of any order are uniformly continuous.

For each $p \in M, d(x, p) \leq d(y, p)+d(x, y)$ for every $x, y \in M$ by the triangle inequality, and similarly with $x$ and $y$ interchanged. This implies that $f_{p}(x)=$ $d(x, p)$ is a real-valued 1-Lipschitz function of order 1. If $0<\alpha \leq 1$ and $r, t$ are nonnegative real numbers, then $\max (r, t) \leq\left(r^{\alpha}+t^{\alpha}\right)^{1 / \alpha}$, which implies that

$$
r+t \leq \max (r, t)^{1-\alpha}\left(r^{\alpha}+t^{\alpha}\right) \leq\left(r^{\alpha}+t^{\alpha}\right)^{1 / \alpha},
$$

or $(r+t)^{\alpha} \leq r^{\alpha}+t^{\alpha}$. It follows that $f_{p, \alpha}=d(x, p)^{\alpha}$ is a 1-Lipschitz function of order $\alpha$ for every $p \in M$ when $\alpha \leq 1$, for the same reasons as for $\alpha=1$.

By contrast, if $f$ is a real or complex-valued Lipschitz function of order $\alpha>1$ on the real line, then $f^{\prime}(x)=0$ for every $x \in \mathbf{R}$, and $f$ is constant. The same argument works on Euclidean spaces of any dimension, but there are metric spaces with nonconstant Lipschitz functions of order $>1$. On the Cantor set there are 
nontrivial locally constant functions, for instance. There are also connected and locally connected snowflake sets with nonconstant Lipschitz functions of order $>1$.

Let $(M, d(x, y))$ be a metric space, and suppose that $a, b$ are real numbers with $a \leq b$ and that $p:[a, b] \rightarrow M$ is a continuous curve in $M$. If $\mathcal{P}=\left\{t_{\ell}\right\}_{\ell=1}^{n}$ is a partition of $[a, b]$, which means that

$$
a=t_{0}<t_{1}<\cdots<t_{n}=b,
$$

then put

$$
\lambda(\mathcal{P})=\sum_{\ell=1}^{n} d\left(p\left(t_{\ell-1}\right), p\left(t_{\ell}\right)\right) .
$$

We say that $p$ has finite length in $M$ if there is an upper bound for $\lambda(\mathcal{P})$ over all partitions $\mathcal{P}$ of $[a, b]$, in which case the length $\lambda$ of $p$ is defined to be the supremum of $\lambda(\mathcal{P})$. This is the same as saying that $p$ has bounded variation when $M$ is $\mathbf{R}$ or $\mathbf{C}$. If $p:[a, b] \rightarrow M$ is a continuous curve of length $\lambda$, then $d(p(a), p(b)) \leq \lambda$. The restriction of $p$ to any subinterval of $[a, b]$ is a continuous curve with length $\leq \lambda$, and hence the diameter of $p([a, b])$ is $\leq \lambda$. Note that a continuous curve has length equal to 0 if and only if it is constant.

Suppose that $\mathcal{P}, \mathcal{P}^{\prime}$ are partitions of $[a, b]$ and that $\mathcal{P}^{\prime}$ is a refinement of $\mathcal{P}$, which is to say that each term in $\mathcal{P}$ is also in $\mathcal{P}^{\prime}$. Using the triangle inequality, one can check that $\lambda(\mathcal{P}) \leq \lambda\left(\mathcal{P}^{\prime}\right)$. As a consequence, it suffices to use partitions of $[a, b]$ that contain a fixed element $r \in[a, b]$ in order to determine the length of $p$. This implies that the length of $p$ on $[a, b]$ is equal to the sum of the lengths of $p$ on $[a, r]$ and on $[r, b]$ for each $r \in[a, b]$. If $p:[a, b] \rightarrow M$ is $C$-Lipschitz of order 1 , then $p$ has finite length $\leq C(b-a)$. Conversely, a continuous path of finite length $\lambda$ can be reparameterized to get a 1-Lipschitz curve on an interval of length equal to $\lambda$. This basically uses the arc-length parameterization of $p$.

If $p:[a, b] \rightarrow M$ is a continuous path of length $\lambda$ and $f$ is a $C$-Lipschitz complex-valued function of order 1 on $M$, then $f \circ p$ is a function of bounded variation on $[a, b]$ of total variation $\leq C \lambda$. If $f$ is locally $C$-Lipschitz of order 1 , then $f \circ p$ is still a function of bounded variation on $[a, b]$ of total variation $\leq C \lambda$, since one can use partitions of $[a, b]$ with small mesh size by passing to suitable refinements. If $f$ is locally $\epsilon$-Lipschitz of order 1 for each $\epsilon>0$, then $f \circ p$ has 
total variation equal to 0 , and $f \circ p$ is constant on $[a, b]$. If $f$ is Lipschitz of order $>1$, then $f$ is locally $\epsilon$-Lipschitz of order 1 for each $\epsilon>0$.

If there is a $k \geq 1$ such that every $x, y \in M$ can be connected by a continuous path of length $\leq k d(x, y)$, and if $f$ is a locally $C$-Lipschitz complex-valued function of order 1 on $M$, then $f$ is $(k C)$-Lipschitz of order 1 . This property holds with $k=1$ when $M$ is a convex set in Euclidean space or a normed vector space more generally, since every pair of elements of $M$ can be connected by a line segment of length equal to the distance between $x$ and $y$. This property also holds for some fractals like the Sierpinski gasket and carpet, for suitable $k>1$. A connected open set $U$ in a normed vector space satisfies this property locally with $k=1$, and every $x, y \in U$ can be connected by a curve of finite length, but the relationship between the lengths of the paths and the distances between $x$ and $y$ may be complicated. Similarly, a connected embedded smooth submanifold of $\mathbf{R}^{n}$ has this property locally with $k$ arbitrarily close to 1 , but otherwise the ambient Euclidean distance may be much smaller than the intrinsic distance on the submanifold, depending on the situation.

\section{Complex-analytic metric spaces}

What might one mean by a "complex-analytic metric space"? Certainly $\mathbf{C}^{n}$ with the standard Euclidean metric ought to be an example, as well as domains in $\mathbf{C}^{n}$ and smooth complex manifolds equipped with suitable geometries, etc.

For a nonstandard example, fix an integer $n \geq 2$, and let $\Sigma_{n}$ be the unit sphere in $\mathbf{C}^{n}$. Thus

$$
\Sigma_{n}=\left\{z \in \mathbf{C}^{n}:|z|=1\right\},
$$

where $|z|=\left(\sum_{j=1}^{n}\left|z_{j}\right|^{2}\right)^{1 / 2}$ for $z=\left(z_{1}, \ldots, z_{n}\right) \in \mathbf{C}^{n}$, as usual. We can think of $\Sigma_{n}$ as a real smooth hypersurface in $\mathbf{C}^{n}$, whose tangent space at $z \in \Sigma_{n}$ is

$$
T_{z} \Sigma_{n}=\left\{v \in \mathbf{C}^{n}: \operatorname{Re} \sum_{j=1}^{n} v_{j} \overline{z_{j}}=0\right\} .
$$

Here Re $a$ denotes the real part of a complex number $a$, and $\bar{a}$ is its complex 
conjugate. Put

$$
C T_{z} \Sigma_{n}=\left\{v \in \mathbf{C}^{n}: \sum_{j=1}^{n} v_{j} \overline{z_{j}}=0\right\}
$$

which is a complex-linear subspace of $\mathbf{C}^{n}$ contained in $T_{z} \Sigma_{n}$. If $\operatorname{Im} a$ is the imaginary part of a complex number $a$, then $C T_{z} \Sigma_{n}$ consists of the $v \in T_{z} \Sigma_{n}$ such that $\operatorname{Im} \sum_{j=1}^{n} v_{j} \overline{z_{j}}=0$. This shows that $C T_{z} \Sigma_{n}$ has real codimension 1 in $T_{z} \Sigma_{n}$, which has real codimension 1 in $\mathbf{C}^{n}$. It is well known that every pair of elements of $\Sigma_{n}$ can be connected by a smooth path $p(t)$ in $\Sigma_{n}$ whose derivative $\dot{p}(t)$ is contained in $C T_{p(t)} \Sigma_{n}$ for every $t$ in the interval on which $p(t)$ is defined. A metric on $\Sigma_{n}$ can be defined using the infimum of the lengths of these paths with a fixed pair of endpoints in $\Sigma_{n}$. With respect to this sub-Riemannian geometry on $\Sigma_{n}, C T_{z} \Sigma_{n}$ is the appropriate tangent space for $\Sigma_{n}$ at $z \in \Sigma_{n}$. By construction, $C T_{z} \Sigma_{n}$ is also a complex vector space in a natural way.

This sub-Riemannian geometry on $\Sigma_{n}$ is compatible with the usual topology, but the corresponding Hausdorff dimension is $2 n$.

On a complex manifold $M$, there is a decomposition of exterior differentiation $d$ into the sum of $\partial$ and $\bar{\partial}$. By definition, a complex-valued function $f$ on an open set $U \subseteq M$ is holomorphic if $\bar{\partial} f=0$ on $U$. On $\Sigma_{n}$, there is an analogous operator $\bar{\partial}_{b}$ based on the complex subspaces of the tangent spaces. The $\bar{\partial}_{b}$ operator is the appropriate $\bar{\partial}$ operator on $\Sigma_{n}$ with respect to the sub-Riemannian geometry.

Similar remarks can be applied to other Cauchy-Riemann manifolds with compatible sub-Riemannian geometries. In order to get a complex-analytic metric space, one ought to have complex structures on the subspaces of the tangent spaces that determine the sub-Riemannian structure. Otherwise, one might have "Cauchy-Riemann sub-Riemannian spaces", with complex structures on subspaces of the subspaces of the tangent spaces that determine the sub-Riemannian structure.

In general, one might ask that a complex-analytic metric space have some sort of tangent spaces, perhaps almost everywhere, and complex structures on these tangent spaces. Some nontrivial holomorphic functions would be nice too.

Of course, there has been a lot of work over the years concerning abstract versions of holomorphic functions on complex spaces, often in terms of algebras 
of continuous functions on topological spaces. An advantage of metric spaces is that there are special classes of functions, like Lipschitz functions and Sobolev spaces when the metric space is equipped with a metric, which are relevant for differentiation and other aspects of analysis. The definition of the tangent spaces of the metric space would normally involve some sort of regular functions and their derivatives.

Geometric measure theory deals extensively with differentiation and tangent spaces for sets that may not be smooth. See $[2,3,62,63,64,65,87,103,104$, $140,141,142]$, for instance, concerning holomorphic chains as currents.

For a metric space equipped with a doubling measure and for which there are suitable versions of Poincaré inequalities, Cheeger [26] has shown that there are versions of classical results on differentiability almost everywhere. This is a very interesting setting in which to consider $\bar{\partial}$ operators.

In particular, one might do this for a space $X$ which is a Cartesian product of an even number of spaces like those described by Laakso [98] and intervals. If $L$ is a Laakso space, then there is a natural projection from the product of a Cantor set $C$ and the unit interval $I$ onto $L$, and another projection from $L$ onto $I$. The composition of these two mappings is the usual coordinate projection from $C \times I$ onto $I$. If a complex structure is defined on $X$ in a compatible way, then one can use these projections onto intervals to get nontrivial holomorphic functions on $X$. One can jazz this up a bit using branching.

One can also look at holomorphic mappings between complex-analytic metric spaces, e.g., nontrivial analytic disks. It is well known that any holomorphic mapping from a disc into the unit sphere $\Sigma_{n}$ in $\mathbf{C}^{n}$ is constant. There are plenty of analytic disks in a product of Laakso spaces and intervals when the complex structure on the product satisfies suitable compatibility conditions.

For that matter, one could view the product of a Cantor set and $\mathbf{C}^{n}$ as a complex-analytic metric space, in which only the complex structure in the $\mathbf{C}^{n}$ directions is employed in the product. Thus a holomorphic function on the product would be holomorphic on each copy of $\mathbf{C}^{n}$, and an analytic disk in the product would be an analytic disk in one of the copies of $\mathbf{C}^{n}$. More precisely, the Cantor set would be treated as not contributing to the tangent space of 
the product or the complex structure. This is consistent with the failure of differentiability theorems for Lipschitz functions on Cantor sets, although one might say instead that the derivative is equal to 0 .

Alternatively, let $E$ be a closed set in $\mathbf{C}^{n}$, and suppose that $f: E \rightarrow \mathbf{C}$ is continuously differentiable in the sense of Whitney. This means that for each $p \in E$ there is a real-linear mapping $d f_{p}: \mathbf{C}^{n} \rightarrow \mathbf{C}$ which is continuous as a function of $p$ and satisfies

$$
f(z)=f(p)+d f_{p}(z-p)+o(1)
$$

uniformly on compact subsets of $E$. The restriction to $E$ of a continuouslydifferentiable function on $\mathbf{C}^{n}$ automatically has this feature, using the ordinary differential of $f$ at $p \in E$. Conversely, a function $f$ on $E$ with this property has an extension to a continuously differentiable function on $\mathbf{C}^{n}$ whose differential at $p \in E$ is equal to $d f_{p}$, by Whitney's extension theorem. If $f$ is a continuouslydifferentiable function on $E$ in the sense of Whitney, then $\bar{\partial} f_{p}$ can be defined using $d f_{p}$ in the usual way, and $\bar{\partial} f_{p}=0$ for every $p \in E$ when $f$ is the restriction to $E$ of a holomorphic function on an open set $U \subseteq \mathbf{C}^{n}$ containing $E$.

If $E$ is a Cantor set or a snowflake, then there are nontrivial functions $f$ on $E$ which are continuously-differentiable in Whitney's sense with $d f_{p}=0$ for every $p \in E$. At the opposite extreme, suppose that $E=\mathbf{R}^{n} \subseteq \mathbf{C}^{n}$ and $f: \mathbf{R}^{n} \rightarrow \mathbf{C}$ is continuously-differentiable as a function on $\mathbf{R}^{n}$. The differential of $f$ at $p \in \mathbf{R}^{n}$ is therefore defined as a real-linear mapping from $\mathbf{R}^{n}$ to $\mathbf{C}$, which has a unique extension to a complex-linear mapping from $\mathbf{C}^{n}$ to $\mathbf{C}$. If we use this extension as $d f_{p}$, then $\bar{\partial} f_{p}=0$.

A basic issue about complex-analytic metric spaces is the strength of the $\bar{\partial}$ operator, starting with the question of whether $|\bar{\partial} f|$ is roughly like $|d f|$ when $f$ is real-valued. This is an elementary feature of the classical case, and there is an analogous statement for Cauchy-Riemann spaces in terms of the tangential part of the differential. However, this does not say much about the strength of the $\bar{\partial}$ operator applied to complex-valued functions, since there are standard local regularity results for holomorphic functions on $\mathbf{C}^{n}$ while the boundary values of holomorphic functions on the unit ball automatically satisfy the tangential Cauchy-Riemann equations on the unit sphere but do not have to be smooth. 
If $f$ is a nice complex-valued function with compact support on $\mathbf{C}^{n}$ and $1<$ $p<\infty$, then

$$
\int_{\mathbf{C}^{n}}|\partial f(z)|^{p} d z \leq A(p, n) \int_{\mathbf{C}^{n}}|\bar{\partial} f(z)|^{p} d z
$$

where $A(p, n)>0$ depends only on $p$ and $n$. This follows from well-known results in harmonic analysis, and there are similar estimates for other norms and spaces of functions. These matters have also been studied extensively for domains in $\mathbf{C}^{n}$, their boundaries, and other complex manifolds and Cauchy-Riemann spaces, with additional terms or boundary conditions, etc., according to the situation. Properties like these are of interest for complex-analytic metric spaces in general, as well as the relationship with a suitable Laplace operator and subharmonicity.

The classical theory of quasiconformal mappings in the plane deals exactly with the Beltrami operators $\bar{\partial}_{\mu}=\bar{\partial}-\mu \partial$ associated to a perturbation of the standard complex structure. The quasiconformality condition $\|\mu\|_{\infty}<1$ ensures that $\left|\partial_{\mu} f\right|$ is comparable to $|d f|$ when $f$ is real-valued. Moreover, it leads to $L^{2}$ estimates for the gradient, and $L^{p}$ estimates when $p$ is sufficiently close to 2 . If a function is holomorphic with respect to $\bar{\partial}_{\mu}$, then it can be expressed as the composition of an ordinary holomorphic function with a quasiconformal mapping with dilatation $\mu$.

It can be easier to make sense of the size $|d f|$ of the differential of a function $f$ on a metric space than the differential $d f$, and it may be easier in some situations to make sense of something like $|\bar{\partial} f|$ than $\bar{\partial} f$. There could also be a decomposition of $|d f|^{2}$ into a sum of parts corresponding to $|\partial f|^{2}$ and $|\bar{\partial} f|^{2}$, analogous to the usual decomposition of $d$ into the sum of $\partial$ and $\bar{\partial}$. One might look at this on the Sierpinski gasket in connection with "analysis on fractals" in the sense of $[86,149,150]$, for instance. The underlying local model for this is the fact that a real-affine function on the plane is uniquely determined by its values on the vertices of a triangle, and the decomposition of a real-linear function into parts that are complex-linear and conjugate-linear. By contrast, this may not work as well for squares and Sierpinski carpets.

Let $(M, d(x, y))$ and $(N, \rho(u, v))$ be metric spaces. A mapping $f: M \rightarrow N$ is said to be Lipschitz if it is Lipschitz of order 1, and it is a bilipschitz embedding of $M$ into $N$ if $\rho(f(x), f(y))$ is bounded from above and below by constant multiples 
of $d(x, y)$ for every $x, y \in M$. For example, the standard embedding of the unit sphere $\Sigma_{n}$ into $\mathbf{C}^{n}$ is bilipschitz with respect to the ordinary Euclidean metric on $\mathbf{C}^{n}$ and the induced Riemannian metric on $\Sigma_{n}$. However, this mapping is Lipschitz and not bilipschitz when one uses the sub-Riemannian geometry on $\Sigma_{n}$ associated to the complex subspaces of the tangent spaces. There are probably a lot of subtleties involved with embeddings of complex-analytic metric spaces.

Note that the boundary values of a holomorphic function on the unit ball in $\mathbf{C}^{2}$ could be considered as a quasiregular mapping from $\Sigma_{2}$ with the usual sub-Riemmanian structure into the complex numbers. Similarly, the standard projection from the product of a Laakso space and an interval or another Laakso space to the complex numbers could also be considered quasiregular. In these examples, the tangent spaces of the domain and range have the same dimension, and quasiregularity can be formulated in terms of the differentials of the mappings as linear transformations between the corresponding tangent spaces. In the first example, the Hausdorff dimension of the domain is strictly larger than the topological dimension, which is strictly larger than the dimension of the tangent spaces. In the second example, the Hausdorff dimension of the domain is strictly larger than the topological dimension, which is equal to the dimension of the tangent spaces. Even for variants of Laakso's construction using Cantor sets with Hausdorff dimension 0 so that the Hausdorff and topological dimensions of the resulting spaces would be the same, the Hausdorff measure would not be $\sigma$ finite in the topological dimension. The fibers of the mapping are at least totally disconnected in the second example, if not discrete. Compare with [71].

\section{Clifford holomorphic functions}

Let $n$ be a positive integer, and let $\mathcal{C}(n)$ be the Clifford algebra over the real numbers $\mathbf{R}$ with $n$ generators $e_{1}, \ldots, e_{n}$. By definition, $\mathcal{C}(n)$ is an associative algebra with a nonzero multiplicative identity element. Thus $\mathcal{C}(n)$ contains a copy of $\mathbf{R}$, and the real number 1 can be identified with the multiplicative identity element of $\mathcal{C}(n)$. The generators $e_{1}, \ldots, e_{n}$ of $\mathcal{C}(n)$ satisfy the relations $e_{l}^{2}=-1$ for $l=1, \ldots, n$, and $e_{q} e_{p}=-e_{p} e_{q}$ when $1 \leq p, q \leq n$ and $p \neq q$. For $I=\left\{l_{1}, \ldots, l_{r}\right\}$, 
$1 \leq l_{1}<l_{2}<\cdots<l_{r} \leq n$, let $e_{I}$ be the element of $\mathcal{C}(n)$ defined by

$$
e_{I}=e_{l_{1}} e_{l_{2}} \cdots e_{l_{r}}
$$

We can include $I=\emptyset$ by putting $e_{\emptyset}=1$. The $2^{n}$ elements $e_{I}$ of $\mathcal{C}(n)$, where $I$ runs through all subsets of $\{1, \ldots, n\}$, forms a basis for $\mathcal{C}(n)$ as a vector space over $\mathbf{R}$.

Actually, one can think of $\mathcal{C}(n)$ as being equal to $\mathbf{R}$ when $n=0$. When $n=1, \mathcal{C}(n)$ is equivalent to the complex numbers $\mathbf{C}$, with the one generator $e_{1}$ corresponding to the complex number $i$. When $n=2, \mathcal{C}(n)$ is equivalent to the quaternions $\mathbf{H}$. Normally one might represent $x \in \mathbf{H}$ as

$$
x=x_{1}+x_{2} i+x_{3} j+x_{4} k,
$$

where $i^{2}=j^{2}=k^{2}=-1, k=i j$, and $j i=-k$, which yield $i k=-j=-k i$ and $j k=i=-k j$. For the identification with $\mathcal{C}(2), i, j \in \mathbf{H}$ correspond to the two generators $e_{1}, e_{2} \in \mathcal{C}(2)$, and $k \in \mathbf{H}$ corresponds to their product $e_{1} e_{2}$.

If $v=\left(v_{1}, \ldots, v_{n}\right) \in \mathbf{R}^{n}$, then we can associate to $v$ the element

$$
\widehat{v}=v_{1} e_{1}+\cdots+v_{n} e_{n}
$$

of $\mathcal{C}(n)$. This defines a linear embedding of $\mathbf{R}^{n}$ into $\mathcal{C}(n)$ such that

$$
\widehat{v}^{2}=-\left(v_{1}^{2}+\cdots+v_{n}^{2}\right)
$$

More generally, if $v=\left(v_{0}, v_{1}, \ldots, v_{n}\right) \in \mathbf{R}^{n+1}$,

$$
\widetilde{v}=v_{0}+v_{1} e_{1}+\cdots+v_{n} e_{n}
$$

and

$$
\widetilde{v}^{*}=v_{0}-v_{1} e_{1}-\cdots-v_{n} e_{n},
$$

then

$$
\widetilde{v} \widetilde{v}^{*}=\widetilde{v}^{*} \widetilde{v}=v_{0}^{2}+v_{1}^{2}+\cdots+v_{n}^{2} .
$$

Similarly, if $x=x_{1}+x_{2} i+x_{3} j+x_{4} k \in \mathbf{H}$, where $x_{1}, x_{2}, x_{3}, x_{4}$ are real numbers, and we put

$$
x^{*}=x_{1}-x_{2} i-x_{3} j-x_{4} k,
$$


then

$$
x x^{*}=x^{*} x=x_{1}^{2}+x_{2}^{2}+x_{3}^{2}+x_{4}^{2} .
$$

If $f$ is a continuously-differentiable function on an open set $U \subseteq \mathbf{R}^{n}$ with values in the Clifford algebra $\mathcal{C}(n)$, then we say that $f$ is left or right Clifford holomorphic if

$$
\sum_{l=1}^{n} e_{l} \frac{\partial f}{\partial x_{l}}=0 \quad \text { or } \quad \sum_{l=1}^{n} \frac{\partial f}{\partial x_{l}} e_{l}=0
$$

on $U$, respectively. Alternatively, let $f$ be a continuously-differentiable function on an open set in $\mathbf{R}^{n+1}$, where $x \in \mathbf{R}^{n+1}$ has components $x_{0}, x_{1}, \ldots, x_{n}$. In this case, we get slightly different versions of Clifford holomorphicity with the equations

$$
\sum_{l=0}^{n} e_{l} \frac{\partial f}{\partial x_{l}}=0, \quad \sum_{l=0}^{n} \frac{\partial f}{\partial x_{l}} e_{l}=0
$$

where $e_{0}=1$. There are also variants of these for the quaternions using $i, j$, and $k$. These are all Generalized Cauchy-Riemann Systems as in [146].

Suppose that $f$ is a continuously-differentiable function on an open set $U$ in $\mathbf{R}^{n}$ with values in $\mathbf{R}^{p}$ for some $p \geq 1$, and that $x$ is an element of $U$ and $v$ is a unit vector in $\mathbf{R}^{n}$. If $f$ satisfies an equation at $x$ like those described in the previous paragraph, then the directional derivative of $f$ at $x$ in the direction of $v$ can be expressed as a linear combination of the directional derivatives of $f$ at $x$ in the directions orthogonal to $v$. For example, if $f$ is a left or right Clifford holomorphic function, then one can check this by multiplying the corresponding differential equation on the left or right by $\widehat{v}$, respectively. Let us say that $f$ is $k$-restricted for some $k \geq 1$ if for every $x \in U$ and every hyperplane $H \subseteq \mathbf{R}^{n}$, the norm of the differential of $f$ at $x$ is less than or equal to $k$ times the norm of the restriction of the differential of $f$ at $x$ to $H$. In each of the cases discussed in the previous paragraph, it follows that $f$ is $k$-restricted for a fixed $k$.

The differential of a real-valued function automatically vanishes on a hyperplane at each point. Hence a real-valued $k$-restricted function on a connected open set is constant. When $p=n=2$, the property of being $k$-restricted is very close to quasiregularity. A key difference is that quasiregularity includes a condition of nonnegative orientation. 
Let us say that a linear mapping $A: \mathbf{R}^{n} \rightarrow \mathbf{R}^{p}$ is $k$-restricted if the norm of $A$ is less than or equal to $k$ times the norm of the restriction of $A$ to any hyperplane in $\mathbf{R}^{n}$. Equivalently, $A$ is $k$-restricted if the norm of $A$ is less than or equal to $k$ times the norm of $A+B$ for every linear mapping $B: \mathbf{R}^{n} \rightarrow \mathbf{R}^{p}$ with rank one. This is also the same as saying that the first singular value of $A$ is less than or equal to $k$ times the second singular value. Thus a continuously-differentiable mapping is $k$-restricted if and only if its differential is $k$-restricted at each point, which is exactly the condition required for the arguments in [146] for improved subharmonicity properties of norms of vector-valued harmonic functions. It follows that $f: U \rightarrow \mathbf{R}^{p}$ is $k$-restricted if and only if the norm of the differential of $f$ at any $x \in U$ is less than or equal to $k$ times the norm of the differential of $f+a \phi$ for every $a \in \mathbf{R}^{p}$ and continuously-differentiable real-valued function $\phi$ on $U$.

As an extension of quaternionic and Clifford analysis, one could replace the usual partial derivatives in the coordinate directions with vector fields with smooth coefficients. The number of vector fields could even be less than the dimension of the space, in which event one might ask that the vector fields satisfy the Hörmander condition that they and their commutators span the tangent space at each point. This would imply that functions with vanishing derivatives in the directions of the vector fields are locally constant in particular. Note that solutions of tangential Cauchy-Riemann equations correspond to special classes of quaternionic and Clifford holomorphic functions associated to suitable vector fields, at least locally, just as for holomorphic functions and quaternionic and Clifford analysis in the classical case.

One can also consider versions of quaternionic and Clifford analysis on metric spaces. Since products of quaternionic or Clifford holomorphic functions are not normally holomorphic even on Euclidean spaces, abstract approaches based on algebras of functions do not work as in the complex case. One might look at $k$-restrictedness of a function $f$ on a metric space in terms of comparing local Lipschitz or Sobolev constants for $f$ with their counterparts for $f+a \phi$ when $a$ is a constant vector and and $\phi$ is real-valued. 


\section{Spaces with Poincaré inequalities}

If $B$ is a ball of radius $R>0$ in $\mathbf{R}^{n}, 1 \leq p<\infty$, and $f$ is a real-valued function on $B$, then

$$
\left(\frac{1}{|B|} \int_{B}\left|f(x)-f_{B}\right| d x\right)^{1 / p} \leq C(n) R\left(\frac{1}{|B|} \int_{B}|\nabla f(x)|^{p} d x\right)^{1 / p},
$$

where $|B|$ denotes the volume of $B$ and $f_{B}$ is the average of $f$ on $B$. One might as well suppose that $f$ is continuously differentiable on $B$, although the inequality also works when $f$ is a locally integrable function on $B$ with distributional first derivatives in $L^{p}(B)$. The limiting case $p=\infty$ corresponds to the statement that a Lipschitz condition is implied by a bound for the gradient.

Juha Heinonen and I posed some questions in [76] about whether suitable versions of these classical Poincaré inequalities on other spaces would imply that the spaces enjoy some sort of approximately Euclidean or sub-Riemannian structure. These questions were answered negatively by remarkable examples of Bourdon and Pajot [20] and Laakso [98]. Perhaps it is better to say that they answered positively the question of whether there could be a lot of spaces of this type. In particular, there are spaces of this type with any Hausdorff dimension greater than or equal to 1, and every such space with at least two elements has Hausdorff dimension greater than or equal to 1 because of connectedness.

I would like to suggest that there are positive results along the lines of the previous questions with additional hypotheses. There is a nice theorem of Berestovskii and Vershik [14] concerning sub-Riemannian geometry of metric spaces under somewhat different conditions, and one may be able to build on their approach. Cheeger's work [26] on differentiability of Lipschitz functions almost everywhere on spaces with Poincaré inequalities ought to be an important step in this direction as well. It may be relatively easy to deal with spaces on which there is sufficient "calculus", and there can be different amounts of structure corresponding to different degrees of calculus.

It can be helpful to look at nilpotent Lie groups and sub-Riemannian spaces more closely in order to understand the general situation better. One can also simply start with a connected smooth manifold $M$ and some smooth vector fields $X_{1}, \ldots, X_{n}$ on $M$ which satisfy the Hörmander condition that the tangent space 
of $M$ at every point is spanned by the $X_{\ell}$ 's and their successive Lie brackets. The smooth functions on $M$ as a smooth manifold would be the same as the smooth functions on $M$ with respect to $X_{1}, \ldots, X_{n}$, but the two structures can measure smoothness in very different ways. A vector field on $M$ is a first-order differential operator in the usual sense but may be considered as an operator of higher order with respect to the $X_{\ell}$ 's. A vector field which can be expressed as the bracket of $r$ of the $X_{\ell}$ 's in some way would typically be considered a differential operator of order $r$ with respect to the $X_{\ell}$ 's. In particular, one might start with a smooth distribution $\mathcal{L}$ of linear subspaces of the tangent spaces of $M$, which contain the $X_{\ell}$ 's and are spanned by them at every point. The Hörmander condition is then a maximal non-integrability condition for $\mathcal{L}$, at least if $\mathcal{L}$ consists of proper subspaces of the tangent spaces of $M$.

However, that brackets of the $X_{\ell}$ 's can be defined at all can be considered as an important integrability condition for the corresponding sub-Riemannian space. To have any nontrivial vector fields on a metric space at all is already quite significant, in the sense of first-order differential operators acting on Lipschitz functions as in [158], for instance. Even if there are a lot of vector fields on metric spaces with Poincaré inequalities by [26], it may not be clear how to deal with their brackets. In the context of complex-analytic metric spaces, it would be interesting to know whether brackets of complex vector fields of $\partial / \partial \bar{z}$ type are of the same type. This is the classical integrability condition for an almost-complex structure on a smooth manifold.

There are classical results about integrating vector fields to get nice mappings on manifolds. Extra compatibility conditions are required on sub-Riemannian spaces to get mappings which respect the geometry in appropriate ways. Even for nilpotent Lie groups with sub-Riemannian structures that are invariant under left or right translations by definition, there may only be finite-dimensional families of mappings with suitable regularity. In some cases there may be no reason for a metric space with Poincaré inequalities to have any nontrivial continuous families of mappings which respect the geometry or the topology. On complex-analytic metric spaces, it would be interesting to consider holomorphic vector fields and the possibility of integrating them to get holomorphic mappings.

As another basis for comparison, suppose that $M$ is a smooth manifold and 
that $V$ is a continuous vector field on $M$ which may not be smooth. There are still results about existence of integral curves for $V$ in $M$, but uniqueness might not hold without more information about the regularity of $V$. Uniqueness can also fail for smooth vector fields on singular spaces. Similarly, let $L$ be a Laakso space, with a projection from the Cartesian product of a Cantor set $C$ and the unit interval $I$ onto $L$. One can follow the standard vector field on $I$ and move in the positive direction at unit speed, and do the same on each parallel copy of $I$ in $C \times I$. Because of the identifications between the copies of $I$ in $L$, one loses uniqueness of the trajectories in $L$. It seems interesting to consider metric spaces with vector fields more broadly, including constructions like Laakso's with different patterns of identifications. One might wish to use probability theory to treat this type of branching, i.e., to follow a vector field with a stochastic process.

On Laakso's and related spaces, there are nice classes of regular functions which are locally equivalent to smooth functions on the unit interval and constant in the direction of the Cantor set. A regular vector field can be defined as a regular function times ordinary differentiation in the direction of the unit interval. A regular vector field applied to a regular function is a regular function, and the bracket of two regular vector fields makes sense and is a regular vector field. Even for regular functions, many of the usual problems are still present. The branching can take place on larger regions.

Suppose now that $M$ is a smooth manifold equipped with some sort of subRiemannian structure. If $V$ is a vector field on $M$ which is smooth with respect to the ordinary smooth structure on $M$, then one can integrate $V$ to get smooth mappings on $M$ which are at least continuous with respect to the sub-Riemannian geometry. If $V$ is admissible for the sub-Riemannian structure, then the integral curves for $V$ are automatically admissible. If $V$ is admissible and $[V, X]$ is admissible when $X$ is, then the mappings on $M$ associated to $V$ are compatible with the sub-Riemannian structure. This is basically a regularity condition for $V$ relative to the sub-Riemannian structure, analogous to the classical Lipschitz condition for the coefficients of a vector field.

Sometimes a vector field is obtained from the gradient of a function. This could be derived from a pairing between functions which includes a pointwise pairing between their gradients, at least implicitly. Such a pairing might be 
positive and symmetric, like a Riemannian metric, or antisymmetric, as for a symplectic structure.

On a Cantor set represented as the Cartesian product of a sequence of finite sets, there are a lot of transformations obtained from permutations in the individual coordinates, including small displacements from permutations in coordinates with large indices. It is not so easy to have nontrivial small displacements on some locally connected spaces, because of intricate topological structure. On spaces with Poincaré inequalities, one can also try to study small displacements in terms of vector fields, perhaps on associated tangent objects. At any rate, it seems interesting to look at group actions on spaces with Poincaré or related inequalities.

I would like to think of a metric space with Poincaré, Sobolev, or similar inequalities as a kind of generalized manifold. One can look at this as a variant of Cantor manifolds [81], which are spaces that are not disconnected by subsets of topological codimension $\geq 2$. This is especially close to isoperimetric inequalities and other estimates of the measure of a set in terms of the size of its boundary.

A lot of analysis related to singular integral operators and classical spaces of functions is available in the vast setting of spaces of homogeneous type [29, 30]. This includes Cantor sets and snowflake spaces, which are important examples with many applications, and which also have nonconstant functions with vanishing gradient. As a next step, one can try to integrate local Lipschitz conditions to estimate the behavior of a function. With Poincaré or Sobolev inequalities, one has stronger forms of calculus involving integrals of derivatives.

Notions of generalized manifolds have been studied extensively in algebraic topology. After all, homology and cohomology are also ways of doing "calculus" on broad classes of spaces. One of the simplest of these notions is that of polyhedral pseudomanifolds. More sophisticated theories deal with intermediate dimensions in the space.

Even for topological manifolds, there can be different types of structures with different versions of calculus. A manifold may be equipped with a smooth structure, for instance, or a piecewise-linear, Lipschitz, or quasiconformal structure more generally. It may be represented as a polyhedron, which might or might 
not have piecewise-linear local coordinates, or coordinates of moderate complexity. Using sub-Riemannian geometry, a manifold can be a fractal and still have Poincaré and Sobolev inequalities.

The apparent irregularities of some spaces with Poincaré or Sobolev inequalities could be attributed to using classical geometry instead of something like noncommutative geometry. With noncommutative geometry, one can try to avoid complicated patchworks of interconnections and gain local or infinitesimal symmetries. Spaces with some version of calculus are basically extensions of smooth manifolds whether they are based on classical or noncommutative geometry, and one might as well try to work with both.

In Connes' theory [32], commutators between singular integral operators and multiplication operators are like derivatives and the Dixmier trace corresponds to integration. The Dixmier trace is an asymptotic version of the trace that applies to slightly more than the usual trace class operators and vanishes on trace class operators. These asymptotic properties are important for localization in the theory. One-dimensional spaces are somewhat exceptional because of unusual regularity of commutator operators.

Of course, one-dimensional sets are exceptional in more classical ways as well. Connectedness plays a large role in this. A nice historical survey related to curvature can be found in the introduction to [123]. Some amazing discoveries about singular integral operators and complex analysis are explained in [112].

At any rate, the general area of analysis on metric spaces has seen a lot of activity, and it seems to me that there is plenty of room for more.

\section{References}

[1] L. Ahlfors, Lectures on Quasiconformal Mappings, 2nd edition, American Mathematical Society, 2006.

[2] H. Alexander, Holomorphic chains and the support hypothesis, J. Amer. Math. Soc. 10 (1997), 123-138. 
[3] F. Almgren, Jr., What can geometric measure theory do for several complex variables?, in Several Complex Variables, 8-21, Princeton University Press, 1993.

[4] L. Ambrosio, Some fine properties of sets of finite perimeter in Ahlfors regular metric measure spaces, Adv. Math. 159 (2001), 51-67.

[5] L. Ambrosio and B. Kirchheim, Currents in metric spaces, Acta Math. 185 (2000), 1-80.

[6] L. Ambrosio and B. Kirchheim, Rectifiable sets in metric and Banach spaces, Math. Ann. 318 (2000), 527-555.

[7] L. Ambrosio and F. Serra Cassano, editors, Lectures Notes on Analysis in Metric Spaces, Scuola Normale Superiore, Pisa, 2000.

[8] L. Ambrosio and P. Tilli, Topics on Analysis in Metric Spaces, Oxford University Press, 2004.

[9] P. Auscher, T. Coulhon, and A. Grigoryan, editors, Heat Kernels and Analysis on Manifolds, Graphs, and Metric Spaces, American Mathematical Society, 2003.

[10] Z. Balogh and M. Rickly, Regularity of convex functions on Heisenberg groups, Ann. Sc. Norm. Sup. Pisa Cl. Sci. (5) 2 (2003), 847-868.

[11] M. Baouendi, P. Ebenfelt, and L. Rothschild, Real Submanifolds in Complex Space and their Mappings, Princeton University Press, 1999.

[12] R. Beals and P. Greiner, Calculus on Heisenberg Manifolds, Princeton University Press, 1988.

[13] A. Bellaïche and J.-J. Risler, editors, Sub-Riemannian Geometry, Birkhäuser, 1996.

[14] V. Berestovskii and A. Vershik, Manifolds with intrinsic metric, and nonholonomic spaces, in Representation Theory and Dynamical Systems, 253267, American Mathematical Society, 1992. 
[15] A. Björn, J. Björn, and N. Shanmugalingam, The Dirichlet problem for $p$ harmonic functions on metric spaces, J. Reine Angew. Math. 556 (2003), 173-203.

[16] A. Björn, J. Björn, and N. Shanmugalingam, The Perron method for $p$ harmonic functions in metric spaces, J. Diff. Eq. 195 (2003), 398-429.

[17] S. Bochner and W. Martin, Several Complex Variables, Princeton University Press, 1948.

[18] A. Boggess, CR Manifolds and the Tangential Cauchy-Riemann Complex, CRC Press, 1991.

[19] M. Bourdon, Immeubles hyperboliques, dimension conforme et rigidité de Mostow, Geom. Funct. Anal. 7 (1997), 245-268.

[20] M. Bourdon and H. Pajot, Poincaré inequalities and quasiconformal structure on the boundary of some hyperbolic buildings, Proc. Amer. Math. Soc. 127 (1999), 2315-2324.

[21] F. Brackx, R. Delanghe, and F. Sommen, Clifford Analysis, Pitman, 1982.

[22] A. Browder, Introduction to Function Algebras, Benjamin, 1969.

[23] L. Capogna, Regularity of quasi-linear equations in the Heisenberg group, Comm. Pure Appl. Math. 50 (1997), 867-889.

[24] L. Capogna and N. Garofalo, Regularity of minimizers of the calculus of variations in Carnot groups via hypoellipticity of systems of Hörmander type, J. Eur. Math. Soc. 5 (2003), 1-40.

[25] L. Capogna and N. Garofalo, Ahlfors type estimates for perimeter measures in Carnot-Carathéodory spaces, J. Geom. Anal. 16 (2006), 457-497.

[26] J. Cheeger, Differentiability of Lipschitz functions on metric measure spaces, Geom. Funct. Anal. 9 (1999), 428-517.

[27] S.-S. Chern, Complex Manifolds without Potential Theory, Springer-Verlag, 1995. 
[28] G. Citti and M. Manfredini, Blow-up in non homogeneous Lie groups and rectifiability, Houston J. Math. 31 (2005), 333-353.

[29] R. Coifman and G. Weiss, Analyse Harmonique Non-Commutative sur Certains Espaces Homogènes: Étude de Certaines Intégrales Singulières, Lecture Notes in Mathematics 242, Springer-Verlag, 1971.

[30] R. Coifman and G. Weiss, Extensions of Hardy spaces and their use in analysis, Bull. Amer. Math. Soc. 83 (1977), 569-645.

[31] D. Cole and S. Pauls, $C^{1}$ Hypersurfaces of the Heisenberg group are $N$ rectifiable, Houston J. Math. 32 (2006), 713-724.

[32] A. Connes, Noncommutative Geometry, based in part on a translation from the French by S. Berberian, Academic Press, 1994.

[33] A. Connes and H. Moscovici, The local index formula in noncommutative geometry, Geom. Funct. Anal. 5 (1995), 174-243.

[34] T. Cummins, A pseudodifferential calculus associated to 3-step nilpotent groups, Comm. Part. Diff. Eq. 14 (1989), 129-171.

[35] D. Danielli, N. Garofalo, and D.-M. Nhieu, Notions of convexity in Carnot groups, Comm. Anal. Geom. 11 (2003), 263-341.

[36] D. Danielli, N. Garofalo, and D.-M. Nhieu, Non-Doubling Ahlfors Measures, Perimeter Measures, and the Characterization of the Trace Spaces of Sobolev Functions in Carnot-Carathéodory Spaces, Mem. Amer. Math. Soc. 857, 2006.

[37] D. Danielli, N. Garofalo, D.-M. Nhieu, and F. Tournier, The theorem of Busemann-Feller-Alexandrov in Carnot groups, Comm. Anal. Geom. 12 (2004), 853-886.

[38] G. David and S. Semmes, Fractured Fractals and Broken Dreams: SelfSimilar Geometry through Metric and Measure, Oxford University Press, 1997.

[39] J. Dixmier, Existence de traces non normales, C. R. Acad. Sci. Paris Sér. A-B 262 (1966), A1107-A1108. 
[40] P. Duren, Theory of $H^{p}$ Spaces, Academic Press, 1970.

[41] L. Evans and R. Gariepy, Measure Theory and Fine Properties of Functions, CRC Press, 1992.

[42] K. Falconer, The Geometry of Fractal Sets, Cambridge University Press, 1985.

[43] H. Federer, Geometric Measure Theory, Springer-Verlag, 1996.

[44] G. Folland and E. Stein, Hardy Spaces on Homogeneous Groups, Princeton University Press, 1982.

[45] B. Franchi, P. Hajłasz, and P. Koskela, Definitions of Sobolev classes on metric spaces, Ann. Inst. Fourier 49 (1999), 1903-1924.

[46] B. Franchi, R. Serapioni, and F. Serra Cassano, Sur les ensembles de périmètre fini dans le groupe de Heisenberg, C. R. Acad. Sci. Paris Sér. I Math. 329 (1999), 183-188.

[47] B. Franchi, R. Serapioni, and F. Serra Cassano, Rectifiability and perimeter in the Heisenberg group, Math. Ann. 321 (2001), 479-531.

[48] B. Franchi, R. Serapioni, and F. Serra Cassano, On the structure of finite perimeter sets in step 2 Carnot groups, J. Geom. Anal. 13 (2003), 421-466.

[49] B. Franchi, R. Serapioni, and F. Serra Cassano, Regular hypersurfaces, intrinsic perimeter and implicit function theorem in Carnot groups, Comm. Anal. Geom. 11 (2003), 909-944.

[50] T. Gamelin, Uniform Algebras, Prentice-Hall, 1969.

[51] T. Gamelin, Uniform Algebras and Jensen Measures, Cambridge University Press, 1978.

[52] J. Garnett, Bounded Analytic Functions, Academic Press, 1981.

[53] N. Garofalo and D.-M. Nhieu, Isoperimetric and Sobolev inequalities for Carnot-Carathéodory spaces and the existence of minimal surfaces, Comm. Pure Appl. Math. 49 (1996), 1081-1144. 
[54] N. Garofalo and F. Tournier, New properties of convex functions in the Heisenberg group, Trans. Amer. Math. Soc. 358 (2006), 2011-2055.

[55] F. Gehring, Topics in quasiconformal mappings, in Proceedings of the International Congress of Mathematicians (Berkeley, 1986), volume 1, 62-80, American Mathematical Society, 1987.

[56] F. Gehring, Quasiconformal mappings in Euclidean spaces, in Handbook of Complex Analysis: Geometric Function Theory, volume 2, 1-29, Elsevier, 2005.

[57] J. Gilbert and M. Murray, Clifford Algebras and Dirac Operators in Harmonic Analysis, Cambridge University Press, 1991.

[58] P. Hajłasz, Sobolev spaces on arbitrary metric space, Pot. Anal. 5 (1996), 403-415.

[59] P. Hajłasz and P. Koskela, Sobolev meets Poincaré, C. R. Acad. Sci. Paris Sér. I Math. 320 (1995), 1211-1215.

[60] P. Hajłasz and P. Koskela, Sobolev Met Poincaré, Mem. Amer. Math. Soc. 688, 2000.

[61] B. Hanson and J. Heinonen, An n-dimensional space that admits a Poincaré inequality but has no manifold points, Proc. Amer. Math. Soc. 128 (2000), 3379-3390.

[62] R. Harvey, Holomorphic chains and their boundaries, in Several Complex Variables, part 1, 309-382, American Mathematical Society, 1977.

[63] R. Harvey and J. King, On the structure of positive currents, Inv. Math. 15 (1972), 47-52.

[64] R. Harvey and B. Lawson, Complex analytic geometry and measure theory, in Geometric Measure Theory and the Calculus of Variations, 261-274, American Mathematical Society, 1986.

[65] R. Harvey and B. Shiffman, A characterization of holomorphic chains, Ann. Math. (2) 99 (1974), 553-587. 
[66] J. Heinonen, Calculus on Carnot groups, Rep. Dept. Math. Stat. 68, University of Jyväskylä, 1995.

[67] J. Heinonen, Lectures on Analysis on Metric Spaces, Springer-Verlag, 2001.

[68] J. Heinonen, Geometric embeddings of metric spaces, Rep. Dept. Math. Stat. 90, University of Jyväskylä, 2003.

[69] J. Heinonen, Lectures on Lipschitz analysis, Rep. Dept. Math. Stat. 100, University of Jyväskylä, 2005.

[70] J. Heinonen, Nonsmooth calculus, Bull. Amer. Math. Soc. (N.S.) 44 (2007), $163-232$.

[71] J. Heinonen and I. Holopainen, Quasiregular maps on Carnot groups, J. Geom. Anal. 7 (1997), 109-148.

[72] J. Heinonen, T. Kilpeläinen, and O. Martio, Nonlinear Potential Theory of Degenerate Elliptic Equations, Oxford University Press, 1993.

[73] J. Heinonen and P. Koskela, Definitions of quasiconformality, Inv. Math. 120 (1995), 61-79.

[74] J. Heinonen and P. Koskela, From local to global in quasiconformal structures, Proc. Nat. Acad. Sci. U.S.A. 93 (1996), 554-556.

[75] J. Heinonen and P. Koskela, Quasiconformal maps in metric spaces with controlled geometry, Acta Math. 181 (1998), 1-61.

[76] J. Heinonen and S. Semmes, Thirty-three yes or no questions about mappings, measures, and metrics, Conform. Geom. Dyn. 1 (1997), 1-12.

[77] K. Hoffman, Banach Spaces of Analytic Functions, Dover, 1988.

[78] L. Hörmander, Hypoelliptic second order differential equations, Acta Math. 119 (1967), 147-171.

[79] L. Hörmander, An Introduction to Complex Analysis in Several Variables, North-Holland, 1973.

[80] L. Hörmander, Notions of Convexity, Birkhäuser, 1994. 
[81] W. Hurewicz and H. Wallman, Dimension Theory, revised edition, Princeton University Press, 1948.

[82] T. Iwaniec and G. Martin, Geometric Function Theory and Non-Linear Analysis, Oxford University Press, 2001.

[83] S. Janson and T. Wolff, Schatten classes and commutators of singular integral operators, Ark. Mat. 20 (1982), 301-310.

[84] D. Jerison, The Poincaré inequality for vector fields satisfying Hörmander's condition, Duke Math. J. 53 (1986), 503-523.

[85] J.-L. Journé, Calderón-Zygmund Operators, Pseudodifferential Operators and the Cauchy Integral of Calderón, Lecture Notes in Mathematics 994, Springer-Verlag, 1993.

[86] J. Kigami, Analysis on Fractals, Cambridge University Press, 2001.

[87] J. King, The currents defined by analytic varieties, Acta Math. 127 (1971), $185-220$.

[88] J. Kinnunen and O. Martio, Nonlinear potential theory on metric spaces, Illinois J. Math. 46 (2002), 857-883.

[89] J. Kinnunen and N. Shanmugalingam, Regularity of quasi-minimizers on metric spaces, Man. Math. 105 (2001), 401-423.

[90] B. Kirchheim, Rectifiable metric spaces: Local structure and regularity of the Hausdorff measure, Proc. Amer. Math. Soc. 121 (1994), 113-123.

[91] B. Kirchheim and V. Magnani, A counterexample to metric differentiability, Proc. Edinburgh Math. Soc. (2) 46 (2003), 221-227.

[92] P. Koosis, Introduction to $H_{p}$ Spaces, 2nd edition, Cambridge University Press, 1998.

[93] A. Koranyi and H. Reimann, Quasiconformal mappings on the Heisenberg group, Inv. Math. 80 (1985), 309-338.

[94] A. Koranyi and H. Reimann, Foundations for the theory of quasiconformal mappings on the Heisenberg group, Adv. Math. 111 (1995), 1-87. 
[95] S. Krantz, A Panorama of Harmonic Analysis, Mathematical Association of America, 1999.

[96] S. Krantz, Function Theory of Several Complex Variables, AMS Chelsea Publishing, 2001.

[97] S. Krantz, Complex Analysis: The Geometric Viewpoint, 2nd edition, Mathematical Association of America, 2004.

[98] T. Laakso, Ahlfors $Q$-regular spaces with arbitrary $Q>1$ admitting weak Poincaré inequality, Geom. Funct. Anal. 10 (2000), 111-123; erratum, 12 (2002), 650 .

[99] T. Laakso, Plane with $A_{\infty}$-weighted metric not bi-Lipschitz embeddable to $\mathbf{R}^{N}$, Bull. London Math. Soc. 34 (2002), 667-676.

[100] M. Lapidus, Analysis on fractals, Laplacians on self-similar sets, noncommutative geometry and spectral dimensions, Top. Met. Nonlinear Anal. 4 (1994), 137-195.

[101] M. Lapidus, Towards a noncommutative fractal geometry? Laplacians and volume measures on fractals, in Harmonic Analysis and Nonlinear Differential Equations, 211-252, American Mathematical Society, 1997.

[102] O. Lehto and K. Virtanen, Quasiconformal Mappings in the Plane, Springer-Verlag, 1973.

[103] P. Lelong, Intégration sur un ensemble analytique complexe, Bull. Soc. Math. France 85 (1957), 239-262.

[104] P. Lelong, Positivity in Complex Spaces and Plurisubharmonic Functions, Queen's University, 1998.

[105] G. Leonardi and S. Rigot, Isoperimetric sets on Carnot groups, Houston J. Math. 29 (2003), 609-637.

[106] G. Lu, J. Manfredi, and B. Stroffolini, Convex functions on the Heisenberg group, Calc. Var. Part. Diff. Eq. 19 (2004), 1-22. 
[107] R. Macías and C. Segovia, Lipschitz functions on spaces of homogeneous type, Adv. Math. 33 (1979), 257-270.

[108] V. Magnani, Elements of Geometric Measure Theory on Sub-Riemannian Groups, Scuola Normale Superiore, Pisa, 2002.

[109] J. Manfredi and B. Stroffolini, A version of the Hopf-Lax formula in the Heisenberg group, Comm. Part. Diff. Eq. 27 (2002), 1139-1159.

[110] P. Mattila, Geometry of Sets and Measures in Euclidean Spaces: Fractals and Rectifiability, Cambridge University Press, 1995.

[111] P. Mattila, Measures with unique tangent measures in metric groups, Math. Scand. 97 (2005), 298-308.

[112] P. Mattila, M. Melnikov, and J. Verdera, The Cauchy integral, analytic capacity, and uniform rectifiability, Ann. Math. (2) 144 (1996), 127-136.

[113] P. Mattila and J. Verdera, Convergence of singular integrals with general measures, J. Eur. Math. Soc. 11 (2009), 257-271.

[114] M. Miranda, Jr., Functions of bounded variation on "good" metric spaces, J. Math. Pures Appl. (9) 82 (2003), 975-1004.

[115] J. Mitchell, On Carnot-Carathéodory metrics, J. Diff. Geom. 21 (1985), $35-45$.

[116] R. Montgomery, A Tour of Subriemannian Geometries, their Geodesics, and Applications, American Mathematical Society, 2002.

[117] F. Morgan, Geometric Measure Theory: A Beginner's Guide, 3rd edition, Academic Press, 2000.

[118] J. Morrow and K. Kodaira, Complex Manifolds, AMS Chelsea, 2006.

[119] A. Nahmod, Geometry of operators and spectral analysis on spaces of homogeneous type, C. R. Acad. Sci. Paris Sér. I Math. 313 (1991), 721-725.

[120] A. Nahmod, Generalized uncertainty principles on spaces of homogeneous type, J. Funct. Anal. 119 (1994), 171-209. 
[121] P. Pansu, Métriques de Carnot-Caratthéodory et quasiisométries des espaces symétriques de rang un, Ann. Math. (2) 129 (1989), 1-60.

[122] S. Pauls, A notion of rectifiability modeled on Carnot groups, Indiana Univ. Math. J. 53 (2004), 49-81.

[123] A. Papadopoulos, Metric Spaces, Convexity and Nonpositive Curvature, European Mathematical Society, 2005.

[124] V. Peller, Hankel Operators and their Applications, Springer-Verlag, 2003.

[125] Y. Reshetnyak, Space Mappings with Bounded Distortion, American Mathematical Society, 1989.

[126] C. Rickart, Natural Function Algebras, Springer-Verlag, 1979.

[127] S. Rickman, Quasiregular Mappings, Springer-Verlag, 1993.

[128] R. Rochberg and S. Semmes, Nearly weakly orthonormal sequences, singular value estimates, and Calderón-Zygmund operators, J. Funct. Anal. 86 (1989), 237-306.

[129] R. Rochberg and S. Semmes, End point results for estimates of singular values of singular integral operators, in Contributions to Operator Theory and its Applications, Birkhäuser, 1988.

[130] W. Rudin, Function Theory in the Unit Ball in $\mathbf{C}^{n}$, Springer-Verlag, 1980.

[131] D. Sarason, Function Theory on the Unit Circle, Virginia Polytechnic Institute and State University, 1978.

[132] C. Selby, An extension and trace theorem for functions of $H$-bounded variation in Carnot groups of step 2, Houston J. Math. 33 (2007), 593-616.

[133] S. Semmes, Mappings and spaces, in Quasiconformal Mappings and Analysis, 347-368, Springer-Verlag, 1998.

[134] S. Semmes, Some Novel Types of Fractal Geometry, Oxford University Press, 2001. 
[135] S. Semmes, An introduction to analysis on metric spaces, Notices Amer. Math. Soc. 50 (2003), 438-443.

[136] S. Semmes, An introduction to Heisenberg groups in analysis and geometry, Notices Amer. Math. Soc. 50 (2003), 640-646.

[137] S. Semmes, Happy fractals and some aspects of analysis on metric spaces, Publ. Mat. 47 (2003), 261-309.

[138] N. Shanmugalingam, Newtonian spaces: An extension of Sobolev spaces to metric measure spaces, Rev. Mat. Iberoamericana 16 (2000), 243-279.

[139] N. Shanmugalingam, Harmonic functions on metric measure spaces, Illinois J. Math. 45 (2001), 1021-1050.

[140] B. Shiffman, Complete characterization of holomorphic chains of codimension one, Math. Ann. 274 (1986), 233-256.

[141] Y.-T. Siu, Analyticity of sets associated to Lelong numbers and the extension of meromorphic maps, Bull. Amer. Math. Soc. 79 (1973), 1200-1205.

[142] Y.-T. Siu, Analyticity of sets associated to Lelong numbers and the extension of closed positive currents, Inv. Math. 27 (1974), 53-156.

[143] N. Stanton, The heat equation in several complex variables, Bull. Amer. Math. Soc. (N.S.) 11 (1984), 65-84.

[144] E. Stein, Singular Integrals and Differentiability Properties of Functions, Princeton University Press, 1970.

[145] E. Stein, Harmonic Analysis: Real-Variable Methods, Orthogonality, and Oscillatory Integrals, with the assistance of T. Murphy, Princeton University Press, 1993.

[146] E. Stein and G. Weiss, Introduction to Fourier Analysis on Euclidean Spaces, Princeton University Press, 1971.

[147] E. Stout, The Theory of Uniform Algebras, Bogden \& Quigley, 1971.

[148] R. Strichartz, Sub-Riemannian geometry, J. Diff. Geom. 24 (1986), 221263; erratum, 30 (1989), 595-596. 
[149] R. Strichartz, Analysis on fractals, Notices Amer. Math. Soc. 46 (1999), 1199-1208.

[150] R. Strichartz, Differential Equations on Fractals: A Tutorial, Princeton University Press, 2006.

[151] F. Trèves, Hypo-Analytic Structures, Princeton University Press, 1992.

[152] J. Väisälä, Lectures on n-Dimensional Quasiconformal Mappings, Lecture Notes in Mathematics 229, Springer-Verlag, 1971.

[153] N. Varopoulos, L. Saloff-Coste, and T. Coulhon, Analysis and Geometry on Groups, Cambridge University Press, 1992.

[154] M. Vuorinen, Conformal Geometry and Quasiregular Mappings, Lecture Notes in Mathematics 1319, Springer-Verlag, 1988.

[155] C. Wang, Subelliptic harmonic maps from Carnot groups, Calc. Var. Part. Diff. Eq. 18 (2003), 95-115.

[156] N. Weaver, Lipschitz algebras and derivations of von Neumann algebras, J. Funct. Anal. 139 (1996), 261-300.

[157] N. Weaver, Lipschitz Algebras, World Scientific, 1999.

[158] N. Weaver, Lipschitz algebras and derivations II, Exterior differentiation, J. Funct. Anal. 178 (2000), 64-112; erratum, 186 (2001), 546.

[159] R. Wells, Differential Analysis on Complex Manifolds, 2nd edition, Springer-Verlag, 1980.

[160] H. Whitney, Complex Analytic Varieties, Addison-Wesley, 1972.

Stephen W. Semmes,

Department of Mathematics,

Rice University,

Houston, Texas, 77251, USA

E-mail: semmes@rice.edu 